\title{
Catching them in Action: Ultrafast Transmission Electron Microscopy
}

\author{
Volkan Ortalan
}

\section{Department of Materials Science and Engineering, University of Connecticut, United States}

Recent developments in instrumentation have made it a very exciting time to perform both fundamental and applied research in the electron microscope. In situ microscopy is moving forward at a rapid pace with the development of gas/liquid stages that permit reaction processes to be imaged and analyzed at atomic resolution. Moreover, the development of nanosecond and faster photoemission electron sources offers the chance to move the high spatial resolution world of electron microscopy into the ultrafast world of materials dynamics. Conventional in situ TEM coupled with ultrafast TEM can be utilized to gain a fundamental understanding of dynamic processes occurring in materials and biological structures. The combination of these capabilities allow for vast improvements of in situ TEM studies limited by video rate in that many processes span multiple time and length scales. Ultrafast dynamic in situ electron microscopy promises to answer challenging questions in the fields ranging from materials science and chemistry to nanoscience and biology.

One of the long-standing challenges in developing fundamental understanding in synthesis and processing of materials is observing the evolution of the structures, in situ. One such example is the synthesis/processing of materials using lasers, which has emerged as a technique that can be used to fabricate complex structures with a high degree of control. ${ }^{1}$ The interaction of the materials subjected to either a pulsed or a continuous laser beam generates extremes of temperature and pressure in the material. ${ }^{2}$ Because of this reason, incorporation of high temporal probing capabilities and lasers to the TEMs has been a critical development required to discover new knowledge about the dynamic evolution of nanostructures under processing conditions on varying time and length scales.

Figure 1 illustrates a schematic of the UTEM in our laboratory at the University of Connecticut with multimode operation (stroboscopic and single-shot) capabilities. High time resolution of the UTEM is achieved by creating short electron pulses that are used to illuminate the specimen. To initiate the laser induced structural and chemical evolution in nanostructures, a sample excitation laser pulse is used. This method is called "pump-probe scheme" and the delay between the two laser pulses, sets the timing of the observation. From the two approaches that enable a time resolution in the range of nanoseconds to femtoseconds, namely stroboscopic ${ }^{3-5}$ and single-shot, ${ }^{6,7}$ the laser-matter interactions in the field of processing of materials requires single-shot technique due to the irreversible nature of the investigated dynamics. Figure 2 represents a set of single-shot experiments performed on Ag nanoparticle $(200 \mathrm{~nm})$ system at two different time delays, namely, $10 \mathrm{~ns}$ and $1400 \mathrm{~ns}$. As can be seen in Fig. 2a, very early dynamics of nanoparticle motions due to the sample excitation laser pulse is observed at $10 \mathrm{~ns}$. At 1400ns, the initial stages of nanoparticle sintering and melting is captured in Fig. $2 b$.

The incorporation of accurate physics for the mechanisms during laser-matter interaction also requires a computational approach that incorporates the atomic scale models to include laser energy absorption, electron-phonon coupling, heat generation/transfer, and structural dynamics. Such links can be investigated using a coupled atomistic-continuum approach by combining classical molecular dynamics simulations with a continuum two-temperature model to model laser energy deposition, heat conduction and phase transformation at the atomic scales. ${ }^{8,9}$ Additionally, multiphysics simulations within the finite element framework to determine the laser-matter interactions (electromagnetics simulations), heat transfer processes, fluid flow and various hydrodynamic effects, ${ }^{10}$ are often critical to obtain a fundamental understanding into the mechanisms associated with the dynamic processes involved during these very 
complex processes. In this presentation, examples of single-shot electron microscopy studies and relevant computational approaches will be presented for selected systems. Additionally, the potential of novel in situ stages for various data acquisition scheme to push the envelope of ultrafast electron microscopy for the investigation of materials will be discussed.

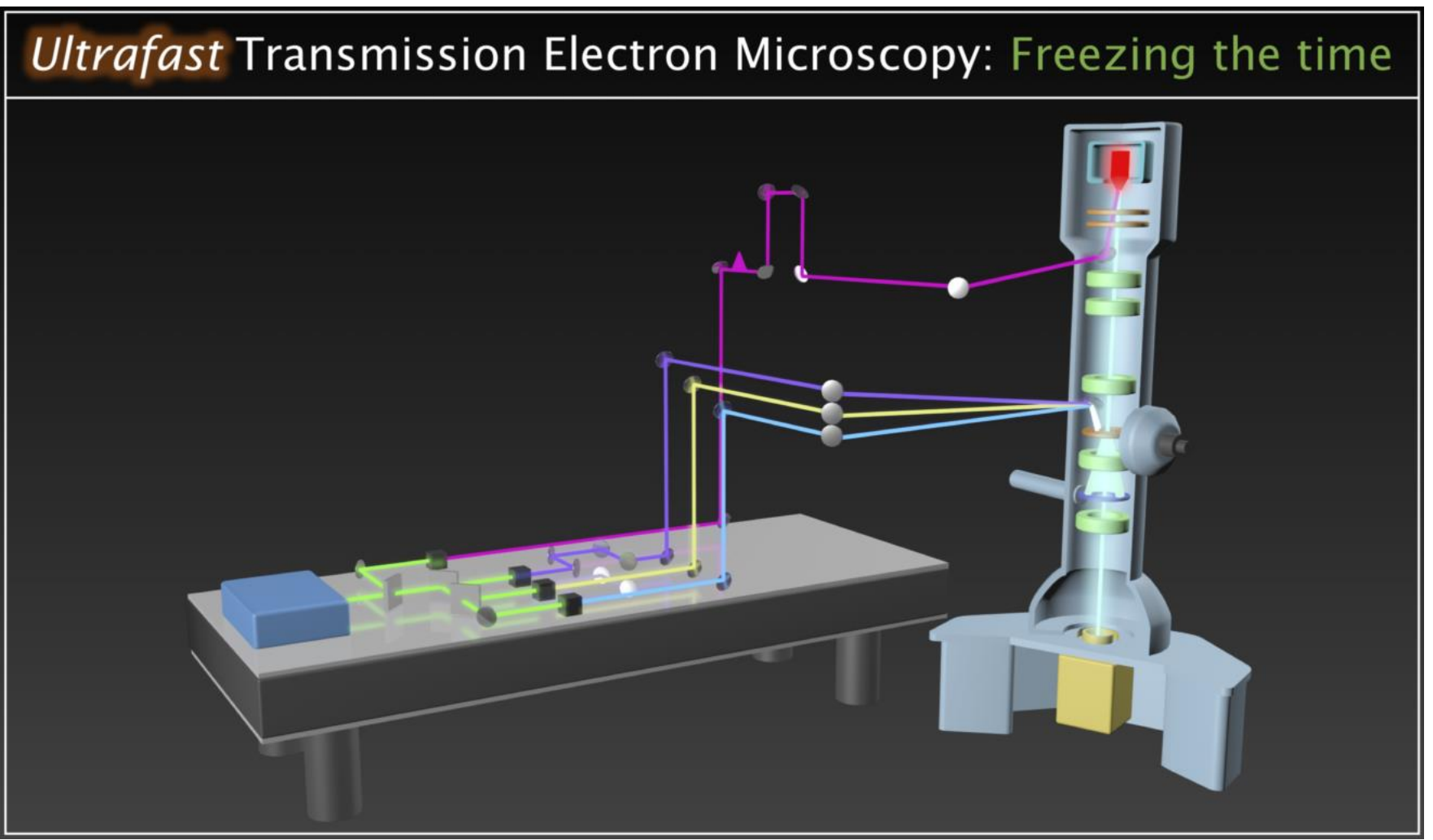

Figure 1. Figure1: Schematic illustration of the Ultrafast TEM in our laboratory at UConn.

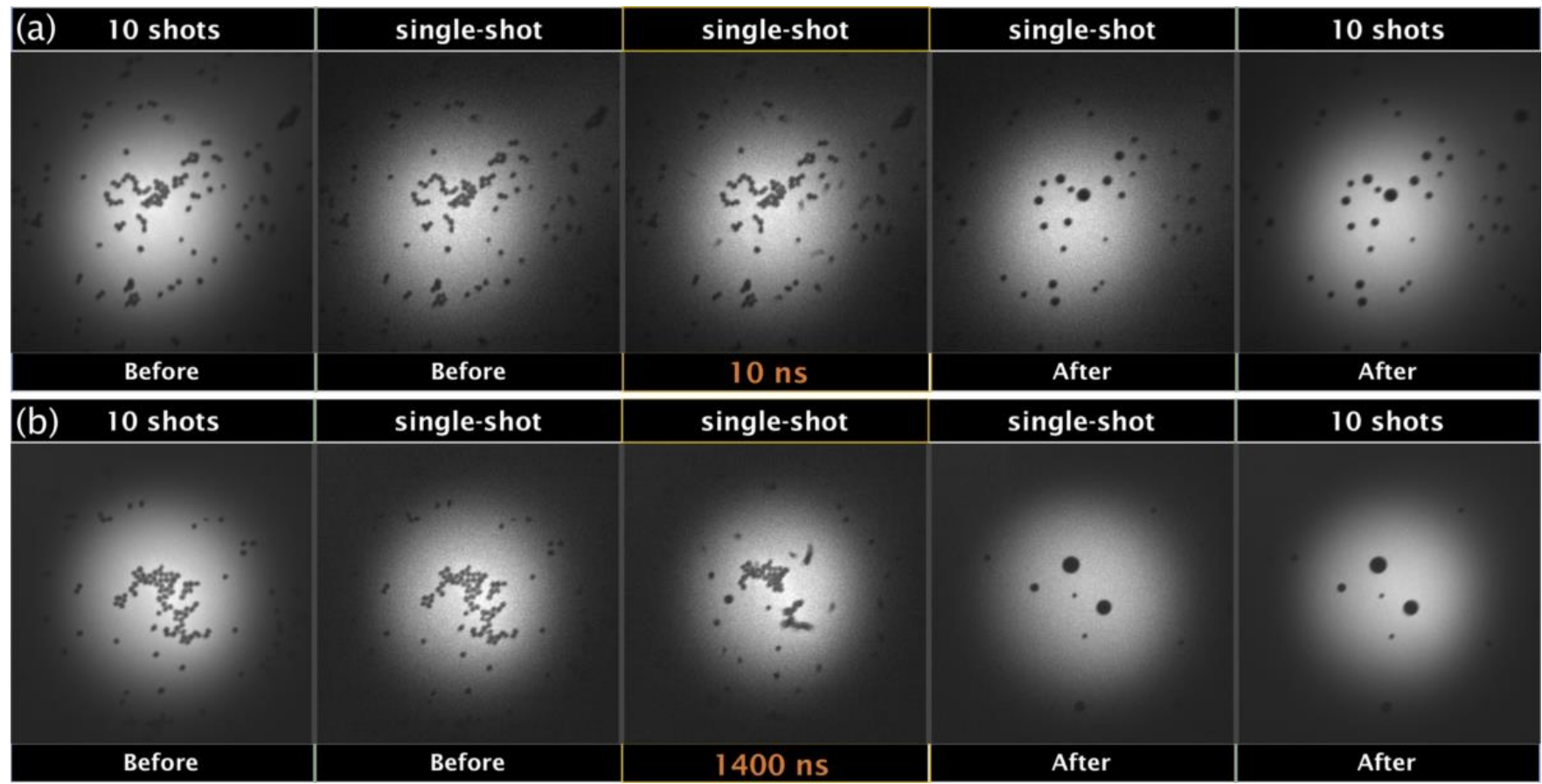


Figure 2. Figure2: Laser induced dynamic evolution of $200 \mathrm{~nm}$ Ag nanoparticle system upon sample excitation laser. (a) $10 \mathrm{~ns}$ time delay and (b) $1400 \mathrm{~ns}$ time delay. Third images in each row are the single shot images showing the transient state of the dynamic process.

References

[1] D. Zhang et. al., "Additive manufacturing of ultrafine-grained high-strength titanium alloys", Nature 576, 91-95 (2019).

[2] S. A. Khairallah et. al., Laser powder-bed fusion additive manufacturing: Physics of complex melt flow and formation mechanisms of pores, spatter, and denudation zones, Acta Mater. 108, 36-45 (2016). [3] B. Barwick, H. S. Park, O.-H. Kwon, J. S. Baskin, A. H. Zewail, 4D Imaging of Transient Structures and Morphologies in Ultrafast Electron Microscopy, Science 322, 1227-1231 (2008).

[4] D. J. Flannigan; P. C. Samartzis; A. Yurtsever; A.H. Zewail, "Nanomechanical Motions of Cantilevers: Direct Imaging in Real-Space and Time with 4D Electron Microscopy". Nano Lett. 9, 875-881 (2009).

[5] V. Ortalan and A. H.Zewail, "4D Scanning Transmission Ultrafast Electron Microscopy: SingleParticle Imaging and Spectroscopy" Journal of the American Chemical Society 133, no. 28 (2011): 10732-10735.

[6] N. D. Browning, G. H. Campbell, J. E. Evans, T. B. LaGrange, B. W. Reed, Electron Microscopy and Spectroscopy on the Ultrafast Timescale, Chemphyschem 11, 781-782 (2010).

[7] G. H. Campbell, T. LaGrange, J. S. Kim, B. W. Reed, N. D. Browning, Quantifying transient states in materials with the dynamic transmission electron microscope, J. Electron Microsc. (Tokyo) 59, S67-S74 (2010).

[8] Ivanov, D. and Zhigilei, L. "Combined Atomistic-Continuum Modeling of Short-Pulse Laser Melting and Disintegration of Metal Films" Physical Review B - Condensed Matter and Materials Physics 68, no. 6 (2003): 433.

[9] Ivanov, D. S. and Zhigilei, L. V. "Combined Atomistic-Continuum Model for Simulation of Laser Interaction with Metals: Application in the Calculation of Melting Thresholds in Ni Targets of Varying Thickness" 79, no. 4-6 (2004): 977-981.

[10] W. E. King et. al., Applied Physics Reviews 2, no. 4 (2015): 041304.

[11] This work was supported by the YIP of Naval Research (CBET-1437219) and MURI Contract No. N00014-16-1-2557. 